\title{
TOTAL BODY RETROGRADE PERFUSION DURING OPERATIONS ON THE DESCENDING THORACIC AORTA
}

Kenzo Yasuura, MD, Yasushi Takagi, MD, Yasutoshi Oohara, MD, and Yoshiyuki Takami, MD, Nagoya, Japan

A left lateral thoracotomy and cannulation of the femoral artery for cardiopulmonary bypass (CPB) are used routinely in operations on the descending thoracic aorta. ${ }^{1}$ Recently, the technique of hypothermic circulatory arrest with retrograde cerebral perfusion proposed by Takamoto and associates ${ }^{2}$ has often been chosen to safely perform an open anastomotic procedure. With the use of this technique, cerebral retrograde perfusion can be achieved by simply elevating the central venous pressure by placing the patient in the Trendelenburg position while the aortic arch is open. The descending thoracic aorta is then occluded with a balloon or a forceps during arterial perfusion in retrograde fashion via a femoral arterial cannula.

To repair the descending thoracic aorta safely, we have developed a circulatory support technique that used the axillary artery as an alternative cannulation site for CPB and the inferior vena cava for total body retrograde perfusion of cold oxygenated blood without occlusion of the descending thoracic aorta.

Technique. After endotracheal intubation with a doublelumen endotracheal tube to permit deflation of the left lung, a cannula is placed in the left radial artery and a catheter in the pulmonary artery for pressure monitoring. The patient is then placed on the right side in a $30^{\circ}$ lateral position. The axillary artery and femoral vein are exposed before thoracotomy. Then, a left anterolateral thoracotomy is done through the fourth intercostal space, the lesion in the thoracic aorta is minimally dissected, and the pericardium is opened anteriorly to the phrenic nerve. After systemic heparinization, a flexible arterial cannula (Sarns/3M Health Care, Ann Arbor, Mich) is threaded into the axillary artery for arterial perfusion, and the femoral vein and the main pulmonary artery are cannulated for venous drainage (Fig 1). For the femoral vein, a multipored long cannula (Medtronic Bio-Medicus, Inc, Eden Prairie, Minn) is introduced into the inferior vena cava and positioned just below the diaphragm through a femoral vein by means of a guide wire technique. Once CPB is established, the left ventricular vent is placed through the left atrial appendage. Systemic cooling is then continued until a nasopharyngeal temperature of $18^{\circ} \mathrm{C}$ is reached. At this point,

From the Department of Thoracic Surgery, Nagoya University School of Medicine, Nagoya, Japan.

Received for publication April 9, 1999; accepted for publication April 27, 1999.

Address for reprints: Kenzo Yasuura, MD, Department of Thoracic Surgery, Nagoya University School of Medicine, Tsurumai-Chou 65, Shouwa-Ku, Nagoya, Japan.

J Thorac Cardiovasc Surg 1999;118:559-61

Copyright $\odot 1999$ by Mosby, Inc.

0022-5223/99 \$8.00+ $0 \quad \mathbf{1 2 / 5 4 / 9 9 5 9 7}$

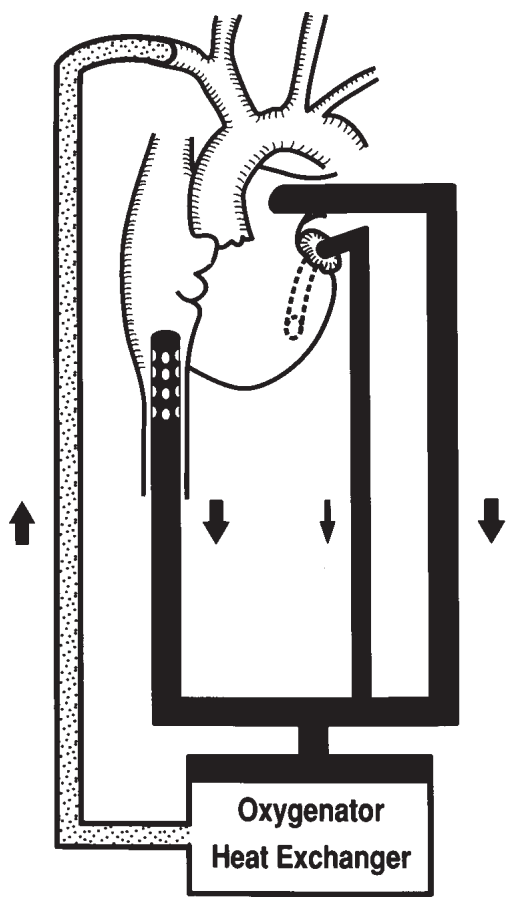

Fig 1. Schema of CPB circuit for operations for thoracic aortic disease.

the patient is placed in the Trendelenburg position, and CPB is transiently discontinued. The venous drainage line from the main pulmonary artery, the left ventricular vent, and the arterial perfusion line are clamped, and the aortic aneurysm is opened. Retrograde perfusion through the inferior vena cava is initiated, maintaining a venous pressure of less than $25 \mathrm{~mm}$ $\mathrm{Hg}$. The aortic aneurysm is resected and a collagen-impregnated vascular graft is anastomosed proximally. During systemic retrograde perfusion, returned blood can be observed in both the proximal and distal segments of the aorta. Cardioplegia is not used because of the relatively short ischemic time. For the purpose of protecting the brain, venous pressure in the superior vena cava and pulmonary artery pressure are carefully monitored. If elevation of mean pulmonary arterial pressure and low pressure in the superior vena cava are recognized, the patient should be placed in a steep Trendelenburg position to securely perfuse the brain. After proximal anastomosis, retrograde perfusion is finished, and antegrade perfusion through the axillary artery is resumed to dispel air and debris for a few minutes. The tube graft is then cut to the appropriate length and clamped to restore perfusion of the upper half of the body. Rewarming is 


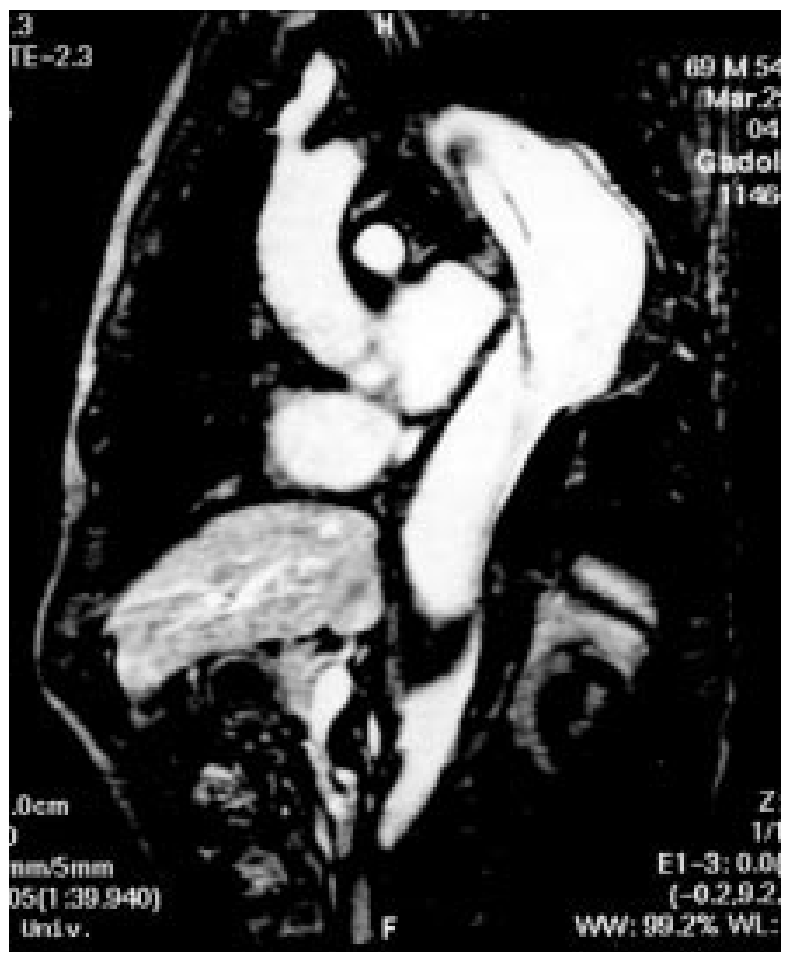

Fig 2. Preoperative magnetic resonance scan showing double lumen of thoracic aorta and intraluminal thrombus in a patient with chronic Stanford type B dissection. Clamping of the aorta and femoral arterial perfusion should be avoided in such a case.

initiated at restoration of antegrade perfusion of the upper part of the body because a distal anastomosis can also be performed through an open procedure without difficulty. When a distal anastomosis is accomplished, the venous drainage tube is transiently clamped to evacuate the air. CPB is totally reestablished and the patient is weaned in the usual manner.

We recently applied this technique in 3 patients who had a distal aortic arch aneurysm and 2 with chronic Stanford type $\mathrm{B}$ dissection. Preoperative evaluation showed the intraluminal thrombus in 2 patients with chronic Stanford type B dissection (Fig 2). All patients underwent replacement of the descending thoracic aorta, and 1 of them had simultaneous reconstruction of the left subclavian artery. The mean CPB time was 152 to 215 minutes (median 176 minutes), retrograde perfusion time was 29 to 37 minutes (median 30 minutes), and complete ischemic time of the lower half of the body was 25 to 43 minutes (median 37 minutes). Flow rate through the inferior vena cava during hypothermic retrograde perfusion was 390 to $790 \mathrm{~mL} / \mathrm{min}$ (median $670 \mathrm{~mL} / \mathrm{min}$ ), and pressure in the superior vena cava was 15 to $18 \mathrm{~mm} \mathrm{Hg}$ (median $16 \mathrm{~mm} \mathrm{Hg}$ ). All patients recovered uneventfully and were discharged to their homes.

Comment. Although the femoral artery is the most common site of arterial cannulation for CPB in the surgical treat-
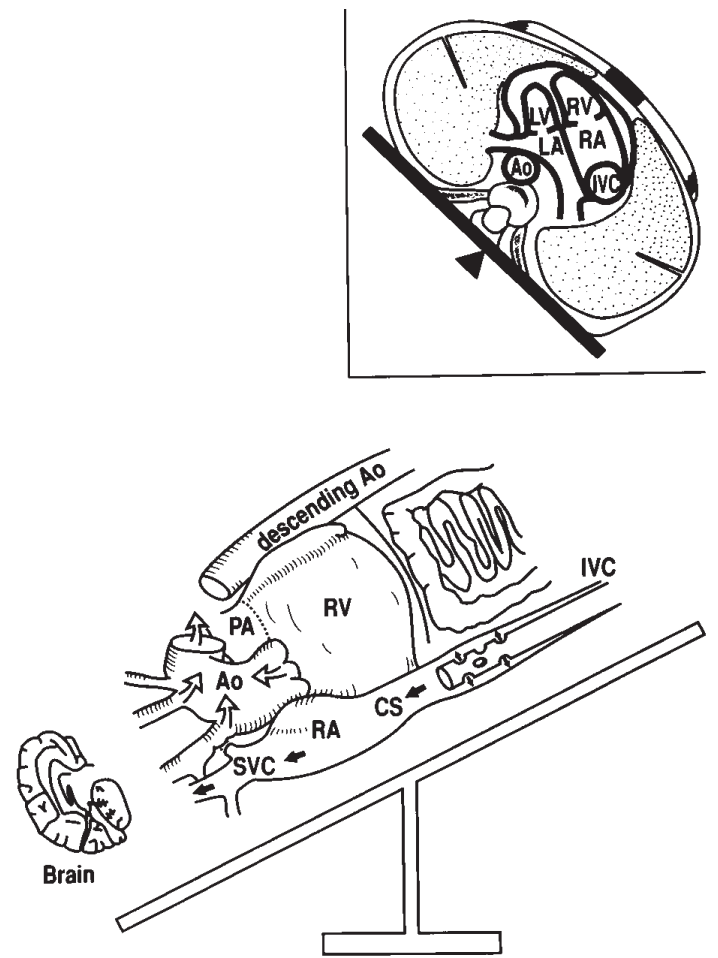

Fig 3. Schema of systemic retrograde perfusion technique. As the inferior vena cava is the lowest in position when the patient's chest is placed in the right lateral decubitus position in the Trendelenburg position, cold oxygenated blood through the cannula in the inferior vena cava is perfused not only to the lower part of the body, but also to the brain. A, Schematic transverse section of heart. B, Positions of the cardiac chambers, aorta, and vena caval system in the chest. Black arrows, Oxygenated blood; white arrows, returned blood. Ao, Aorta; $P A$, pulmonary artery; $R A$, right atrium; $R V$, right ventricle; $S V C$, superior vena cava; $I V C$, inferior vena cava; $C S$, coronary sinus; $L A$, left atrium; $L V$, left ventricle.

ment of descending thoracic aortic aneurysms, this technique has a disadvantage of severe neurologic injury caused by embolism and/or malperfusion resulting from retrograde aortic perfusion through the femoral artery. ${ }^{3}$ Accordingly, the axillary artery is recommended as an alternative cannulation site for antegrade aortic perfusion. ${ }^{4}$

For the repair of descending thoracic aortic disease, with respect to protecting the brain, Takamoto and associates ${ }^{2}$ proposed a hypothermic retrograde cerebral perfusion technique by simply elevating the central venous pressure by using the Trendelenburg position with occlusion of the descending thoracic aorta. This technique makes it possible to have a longer circulatory arrest time than that obtained by hypothermic circulatory arrest alone. However, their technique requires femoral artery cannulation for arterial perfusion and always requires occlusion of the descending thoracic aorta with a balloon or a forceps to perfuse the brain and to evacuate air. 
Furthermore, as venous blood that returned from the lower half of the body via the femoral artery retrogradely perfuses the brain, it remains unclear whether the oxygen level in the venous blood, which may depend on the metabolic state of the visceral organs, can adequately meet the demands of the brain tissue.

In the surgical treatment of complex aortic disease, it is important to avoid complications related to the diseased aorta and to reduce the potential for neurologic injury. We have devised the technique of total body retrograde perfusion using axillary artery cannulation, which does not require occlusion of the distal descending aorta for the purpose of perfusing the brain and precluding the suction of air. When the long multipored cannula is positioned near the diaphragm, the continuous retrograde perfusion through the multipored cannula at this site can perfuse the brain and the visceral organs simply by adjusting the operating table and precludes air suction in the lower half of the body. As the vena caval system is located lower than the right ventricle, main pulmonary artery, and aortic arch, the retrograde flow can be determined and adjusted by monitoring both the pulmonary artery pressure and the pressure in the superior vena cava (Fig 3).
In contrast to the technique proposed by Takamoto and associates, ${ }^{2}$ our method can achieve retrograde perfusion of the brain with adequately oxygenated blood, in addition to minimizing the risk of debris emboli to the brain and other important visceral organs. This modified technique is a safer alternative for operations on the descending thoracic aorta.

\section{REFERENCES}

1. Spentpetery S, Crisler C, Grinnan GLB. Deep hypothermic arrest and left thoracotomy for repair of difficult thoracic aneurysms. Ann Thorac Surg 1993;55:830-3.

2. Takamoto S, Okita Y, Ando M, Morota T, Handa N, Kawashima Y. Retrograde cerebral circulation for distal aortic arch surgery through a left thoracotomy. J Card Surg 1994;9: 576-83.

3. Westaby S, Katsumata T. Proximal aortic perfusion for complex arch and descending aortic disease. J Thorac Cardiovasc Surg 1998;115:162-7.

4. Sabik JF, Lytle BW, McCarthy PM, Cosgrove DM. Axillary artery: an alternative site of arterial cannulation for patients with extensive aortic and peripheral vascular disease. J Thorac Cardiovasc Surg 1995;109:885-91.

\title{
THYMOMA ENCASING LAST PATENT VEN GRAFT TO THE HEART
}

\author{
Michael T. Jaklitsch, MD, John G. Byrne, MD, and Carlos Mery, Boston, Mass, and Monterrey, Mexico
}

A 64-year-old man had progressive weakness 11 years after coronary artery bypass surgery. In 1987, he received 4 vein grafts to the left anterior descending (LAD), the first obtuse marginal, the ramus, and the posterior descending arteries after a myocardial infarction. During the summer of 1998, he began having progressive ptosis, slurred speech, fatigue, and myalgias. Serum anti-acetylcholine-receptor antibodies and an edrophonium test confirmed the clinical diagnosis of myasthenia gravis. Pyridostigmine, $60 \mathrm{mg} 3$ times a day, produced symptomatic improvement.

A chest computed tomographic scan identified a $3.5-\mathrm{cm}$ homogeneous anterior mediastinal mass anterior to the pul-

From the Divisions of Thoracic Surgery and Cardiac Surgery, Brigham and Women's Hospital, Harvard Medical School, Boston, Mass, and ITESM Medical School, Monterrey, Mexico.

Received for publication May 3, 1999; accepted for publication May 7, 1999.

Address for reprints: Michael T. Jaklitsch, MD, Division of Thoracic Surgery, Brigham and Women's Hospital, 75 Francis St, Boston, MA 02115 .

J Thorac Cardiovasc Surg 1999;118:561-3

Copyright (C) 1999 by Mosby, Inc.

$0022-5223 / 99 \$ 8.00+0 \quad \mathbf{1 2 / 5 4 / 9 9 9 3 1}$ monary artery consistent with a thymoma (Fig 1). A partially calcified vein graft was contiguous with the tumor.

Coronary angiography revealed severe native vessel disease (90\% left main, $100 \%$ proximal LAD, $100 \%$ first obtuse marginal, and $100 \%$ proximal right coronary artery) with complete occlusion of 3 of the 4 grafts (first obtuse marginal, ramus, and posterior descending artery). The LAD graft, which appeared to pass through the tumor, was the only patent vessel to the coronary circulation. Left ventriculography showed no wall motion abnormalities and an ejection fraction of 0.67 .

We prepared to remove the only patent vessel providing coronary circulation along with the tumor. The left saphenous vein was harvested. The patient was heparinized and cardiopulmonary bypass (CPB) was established via the left femoral artery and vein before the chest incision. A redo sternotomy was performed without complications and a $4.2-\mathrm{cm}$ upper anterior mediastinal mass was exposed. Manipulation of the sternum was minimized until both pleura were opened wide. The thymoma was attached to the pericardium and the pulmonary artery conus where the pericardium had been opened in 1987. We observed $1-\mathrm{cm}$ erosion into the left pleural space anterior to the hilum without evidence of drop metastases. 